\title{
Actividad de la hormiga Linepithema dispertitum (Hymenoptera: Formicidae) a lo largo del día y en diferentes estados de inflorescencia de Heliconia rostrata (Heliconiaceae)
}

\author{
Pablo R. Gutiérrez-Martínez \\ Escuela de Biología, Universidad de Costa Rica, 2060 San José, Costa Rica; pablorgmbiol@hotmail.com
}

Recibido 13-VII-2012 Corregido 1-X-2012 Aceptado 6-XI-2012

\begin{abstract}
Activity of the ant Linepithema dispertitum (Hymenoptera: Formicidae) at different times of day and in different stages of inflorescence of Heliconia rostrata (Heliconiaceae). Ants are eusocial insects found in most zoogeographic areas and are of great relevance in trophic interactions. This study was conducted in Costa Rica with a colony of Linepithema dispertitum at the base of the pseudostem of Heliconia rostrata. In order to understand the activity of the ants at different times of day, their abundance during five different stages of inflorescence and to learn more about the interactions between plants and ants, two replicates of 255 observations were carried out. Ants were counted in inflorescences from 6:00h to 24:00h, corresponding in each replicate to 85 counts at all times of day, and with 51 counts in each stage of inflorescence; to analyze the data the Kruskal-Wallis nonparametric test was used. Both replicates, despite some differences, share several characteristics: the average number of ants found in the inflorescences in each moment of the day was different in the morning (being the lowest), afternoon (intermediate), and evening (being the highest). Linepithema dispertitum was least abundant in the closed inflorescences and those with withered bracts, on the other hand, the highest averages were in open inflorescences that not wilted and when there was greater availability of food.
\end{abstract}

KEY WORDS

Trophic association, bracts, inflorescence, Linepithema dispertitum, nectar.

\section{RESUMEN}

Las hormigas son insectos eusociales, se encuentran en la mayoría de zonas zoogeográficas y son muy importantes en una serie de interacciones tróficas. El estudio se llevó a cabo en Costa Rica con una colonia de Linepithema dispertitum en la base de los pseudotallos de Heliconia rostrata. Con el propósito de conocer la relación y la actividad de la colonia en distintos momentos del día, su preferencia ante cinco estados de inflorescencia y conocer más sobre las interacciones entre la planta y la hormiga; se hicieron dos repeticiones de 255 conteos de hormigas presentes en inflorescencias de las 6:00h a las 24:00h, correspondiendo en cada repetición a 85 conteos en cada momento del día, y con 51 conteos en cada estado de inflorescencia. Para analizar los datos se realizaron pruebas no paramétricas de Kruskall-Wallis. Ambas repeticiones a pesar de tener diferencias significativas comparten características: los promedios de hormigas en las inflorescencias en cada momento del día fueron significativamente diferentes, por la mañana (el más bajo), la tarde (intermedio) y la noche (el más alto). Linepithema dispertitum además tuvo diferencias significativas en los estados de inflorescencia que visita, menos abundante en las inflorescencias cerradas y las que tienen brácteas marchitas; por otro lado los promedios más altos estaban en inflorescencias abiertas y sin brácteas marchitas, por tanto en las que había mayor disponibilidad de alimento.

\section{PALABRAS CLAVE}

Asociación trófica, brácteas, inflorescencia, Linepithema dispertitum, néctar.
Las hormigas son insectos eusociales, con gran diversidad, tanto funcional como taxonómica (Alatorre-Bracamontes \& Vásquez-Bolaños, 2010). Se encuentran en todas las regiones zoogeografícas del mundo, están entre los organismos que dominan la tierra, con una biomasa del entorno del 10 al 15\% del total de la biomasa animal de la mayoría de los ecosistemas, son muy importantes interviniendo en el ciclo de nutrientes en la naturaleza, en el enriquecimiento de los suelos y en una gran diversidad de interacciones tróficas (Redolfi, Tinaut, Pascual \& Campos, 2003). Existe evidencia de que las hormigas nectívoras establecen interacciones con plantas de las cuales obtienen su alimento, muchas veces constituyen en una defensa contra posibles amenazas, estas relaciones involucran 
desde sistemas obligados hasta aquellos con relaciones difusas (Farji-Brener, Folgarait \& Protomastro, 1992).

Las heliconias son plantas de gran interés para los insectos que son atraídos por el néctar de sus flores, las inflorescencias miden entre 35 a $50 \mathrm{~cm}$ de longitud, están formadas por un péndulo y estructuras modificadas llamadas brácteas, dentro de cada bráctea hay un número variable de flores hermafroditas, la planta se beneficia de los nectívoros, generalmente polinizadas por colibrís (Jerez, 2007). En Heliconia imbricata se ha observado que el volumen de néctar secretado puede ser mayor al incrementarse su consumo; en otros casos se ha documentado que hay diferencias en la producción de néctar entre las flores de diferente edad y de forma general entre flores de una misma planta; las características del néctar pueden variar con la hora del día y con el momento de floración (Guitián, Navarro \& Guitián, 1995).

Las hormigas no eligen los recursos alimenticios al azar, estas emplean una conducta selectiva y una mayor actividad en los recursos preferidos en cuanto a calidad y cantidad (Farji-Brener et al., 1992). Por otro lado estudios en hormigas ha revelado que la actividad de las colonias varia durante el día y esos cambios dependen en gran medida de su comportamiento como especie (Redolfi et al., 2003).

El propósito de esta investigación es identificar una hormiga que se alimenta del néctar de inflorescencias de Heliconia rostrata, además se desea saber si existen preferencias en cuanto al estado de inflorescencia del que obtienen su alimento; también conocer si existen cambios en la actividad de la hormiga en distintos momentos del día. El estudio de la actividad y preferencias alimenticias de Linepithema dispertitum es de importancia básica para trabajos posteriores sobre interacciones tróficas con especies en su entorno, y conocer más acerca del comportamiento de las hormigas; por otra parte estudios similares no han sido realizados antes en Costa Rica.

\section{METODOLOGÍA}

El estudio se llevo a cabo en un jardín ubicado en Esquipulas en Palmares de Alajuela, Costa Rica $\left(10^{\circ} 3^{\prime} 31,45^{\prime \prime} \mathrm{N}\right.$, $84^{\circ} 25^{\prime} 9,36^{\prime \prime} \mathrm{O}$; altitud $\left.1005 \mathrm{msnm}\right)$. Se localizó una colonia de hormigas en la base de los pseudotallos de Heliconia rostrata. La especie de hormiga se determinó con ayuda de la clave de Wild (2007); posteriormente, por medio de fotografías y correo electrónico, el autor de la clave confirmo la determinación.

Para determinar la actividad de la hormiga en distintos momentos del día se realizaron observaciones en tres momentos: mañana (6:00h a 12:00h), tarde (12:00h a 18:00h) y noche (18:00h a 24:00h), además de su preferencia por el estado de inflorescencia en Heliconia rostrata: estado 1 (brácteas cerradas), estado 2 (con $\geq 1<6$ de brácteas abiertas), estado 3 (con $\geq 6<12$ de brácteas abiertas), estado 4 (con $\geq 12<18$ de brácteas abiertas), y el estado 5 (con $\geq 18$ de brácteas abiertas y marchitas); se contaron las hormigas presentes en las inflorescencias de las 6:00 h a las 24:00h en intervalos de tiempo de 15 minutos, se hicieron dos repeticiones de 255 observaciones cada una, la primera el 10 de mayo del 2012, la siguiente el 17 de mayo del 2012, para cada repetición, se eligieron al azar 17 intervalos de tiempo de 24 posibles para cada estado de inflorescencia en cada momento del día; se hicieron 85 conteos en cada momento del día, con 51 conteos en cada estado de inflorescencia para el final de las observaciones. Se hicieron pruebas no paramétricas de KruskallWallis con el fin de detectar diferencias significativas en las repeticiones para los momentos del día y en cada estado de inflorescencia, el análisis se hizo mediante Excel 2007 y el programa estadístico JMP (SAS Institute Inc 2007), de este último se obtuvo el rango promedio (Score Mean) en ambas repeticiones, para cada momento del día y cada estado de inflorescencia; el valor absoluto de la diferencia entre cada par de rangos (|Ri-Rj|) se comparo con el valor critico de Kruskall-Wallis (Ck), con diferencias significativas en aquellos $|\mathrm{Ri}-\mathrm{Rj}|$ con valores mayores o igual al Ck.

\section{RESULTADOS}

La hormiga se determinó como Linepithema dispertitum. En este estudio se observó que a pesar de alimentarse del néctar de Heliconia rostrata la colonia no mostró una actitud defensiva hacia ésta, ni se comportó de forma agresiva en presencia de intrusos; además se registró que la hormiga se asocia con insectos de la familia Pseudococcidae, esto solo en inflorescencias con brácteas marchitas.

Las repeticiones tuvieron diferencias significativas $(N=510 ; p<0,05 ; \beta=1)$, la primera con un promedio de 7,56 y en la segunda de 9,24 hormigas. La primera repetición tuvo diferencias significativas ( $N=255 ; p<0,05 ; \beta=1)$ en los momentos del día, los tres diferentes entre sí, la mañana tuvo el promedio más bajo de hormigas, seguido de la tarde y la noche el más alto; los promedio de hormigas presentes en los estados de inflorescencia tuvieron diferencias significativas ( $N=255 ; p<0,05 ; \beta=0,92$ ), el estado 1 y 5 no tuvieron diferencias significativas entre sí, con los promedios más bajos; por otro lado los estados 2, 3 y 4 sin diferencias significativas entre sí, con los promedios más altos (Cuadro 1 y 2).

La segunda repetición también mostró diferencias significativas en los momentos del día $(N=255 ; p<0,05 ; \beta=1)$, 
los tres diferentes entre sí, la mañana tuvo el promedio más bajo, seguido de la tarde y la noche el más alto. Los promedios de las hormigas presentes en los estados de inflorescencia tuvieron diferencias significativas ( $\mathrm{N}=255$; $p<0,05 ; \beta=0,99$ ), los estados 1,2 y 5 sin diferencias significativas entre sí y los promedios más bajos; el estado 3 con un promedio significativamente distinto y actividad intermedia; por otro lado el estado 4 con el promedio más alto de hormigas y significativamente distinto (Cuadro 1 y 2). La actividad de las hormigas en la planta en las dos repeticiones tuvo su punto más bajo en la mañana entre las 6:00h y las 7:00h, con su punto más alto en la tarde entre 19:00h y las 20:00h (Fig. 1).

\section{DISCUSIÓN}

Linepithema dispertitum es una hormiga de bosque de montaña, pero también se ha registrado en una amplia gama de hábitats, ha sido recolectada de 130 a más de $3000 \mathrm{~m}$ de altitud, se encuentra distribuida del norte de México hasta Panamá, con una población aislada por encima de los 2 400m en la Cordillera Central de la Española; en Costa Rica hay un registro en San Gerardo por Phill Ward en 1985 (Wild, 2007). Por otro lado en Colombia se han documentado colonias de Linepithema dispertitum asociadas a Heliconia stricta y Heliconia platystachys (Ramírez, Chacon, Armbrecht \& Z. Calle, 2001).
CUADRO 1

Promedio y desviación estándar de hormigas en los estados de inflorescencia y momentos del día, entre paréntesis el rango promedio de la prueba Kruskall-Wallis

\begin{tabular}{lcc}
\hline \multirow{2}{*}{ Variable } & \multicolumn{2}{c}{ Repeticiones } \\
\cline { 2 - 3 } Mañana & $(82,48)$ & $(78,69)$ \\
& $3,08 \pm 4,63$ & $4,45 \pm 3,84$ \\
Tarde & $(117,3)$ & $(123,2)$ \\
Noche & $5,49 \pm 6,20$ & $8,33 \pm 7,12$ \\
Estado 1 & $(184,3)$ & $(182,1)$ \\
Estado 2 & $14,1 \pm 10,40$ & $14,9 \pm 8,23$ \\
& $(95,83)$ & $(96,98)$ \\
Estado 3 & $3,53 \pm 3,86$ & $5,68 \pm 4,37$ \\
& $(140,3)$ & $(98,19)$ \\
Estado 4 & $9,75 \pm 6,57$ & $5,96 \pm 5,13$ \\
& $(146,4)$ & $(142,1)$ \\
Estado 5 & $11,7 \pm 13,46$ & $10,3 \pm 7,48$ \\
& $(110,3)$ & $(194,8)$ \\
& $5,49 \pm 7,43$ & $7,08 \pm 6,25$ \\
& & $(107,9)$
\end{tabular}

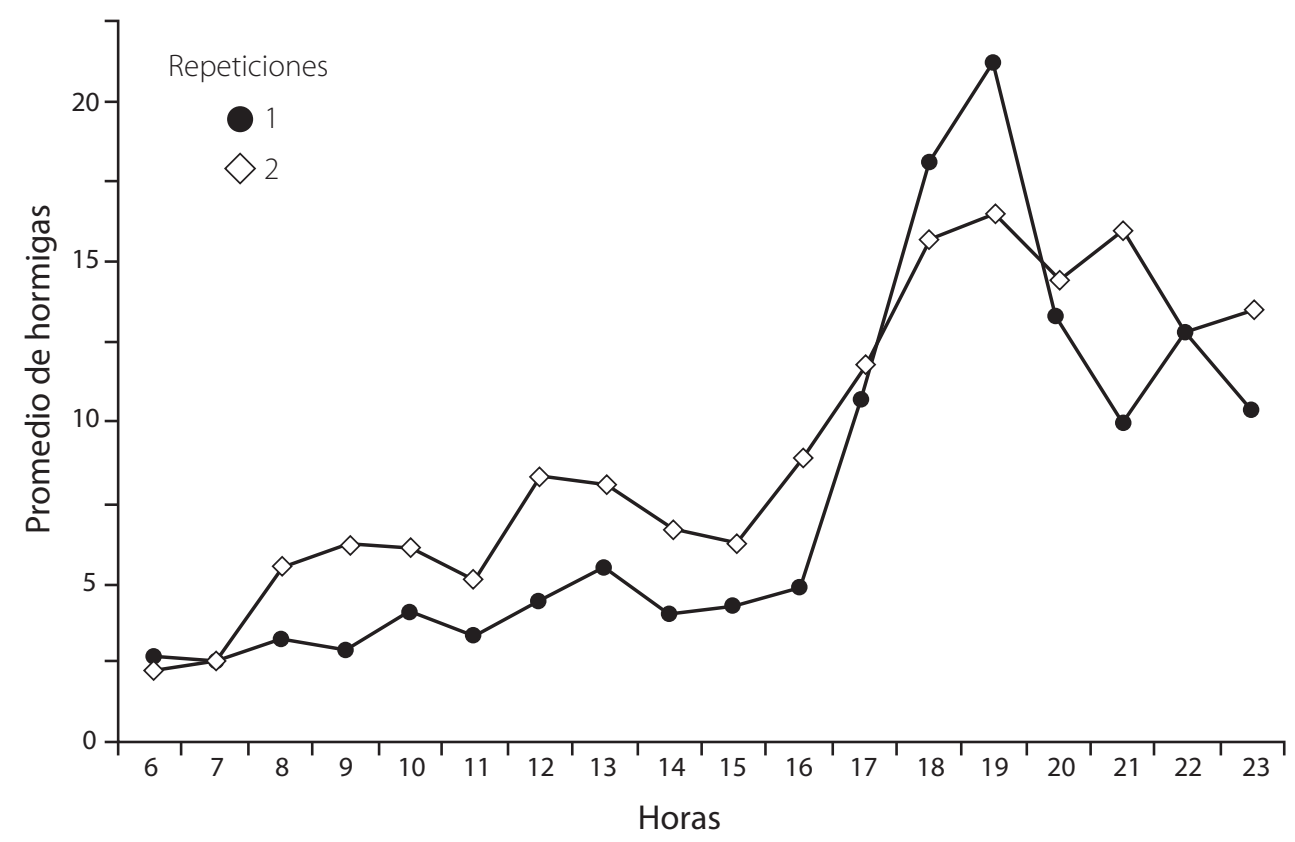

FIG.1. Actividad de Linepithema dispertitum en la mañana (6:00h a 12:00h), tarde (12:00h a 18:00h) y noche (18:00h a 24:00h) en inflorescencias de Heliconia rostrata. 
CUADRO 2

Análisis no paramétricos de Kruskall-Wallis para ver diferencias significativas en los momentos del día y condiciones de inflorescencia

\begin{tabular}{|c|c|c|c|c|}
\hline Comparaciones & $\mathbf{n}$ & $|\mathrm{Ri}-\mathrm{Rj}|$ & Ck & Significancia \\
\hline repetición 1 vs repetición 2 & 255 & 56,39 & 17,29 & Significativa \\
\hline mañana vs tarde (repetición 1) & 85 & 34,82 & 14,51 & Significativa \\
\hline mañana vs noche (repetición 1) & 85 & 101,8 & 14,51 & Significativa \\
\hline tarde vs noche (repetición 1) & 85 & 67,03 & 14,51 & Significativa \\
\hline mañana vs tarde (repetición 2) & 85 & 44,53 & 14,51 & Significativa \\
\hline mañana vs noche (repetición 2) & 85 & 103,4 & 14,51 & Significativa \\
\hline tarde vs noche (repetición 2) & 85 & 58,86 & 14,51 & Significativa \\
\hline estado 1 vs estado 2 (repetición 1) & 51 & 44,46 & 18,73 & Significativa \\
\hline estado 1 vs estado 3 (repetición 1) & 51 & 50,52 & 18,73 & Significativa \\
\hline estado 1 vs estado 4 (repetición 1) & 51 & 51,37 & 18,73 & Significativa \\
\hline estado 1 vs estado 5 (repetición 1) & 51 & 14,48 & 18,73 & NS \\
\hline estado 2 vs estado 3(repetición 1) & 51 & 6,059 & 18,73 & NS \\
\hline estado 2 vs estado 4 (repetición 1) & 51 & 6,912 & 18,73 & NS \\
\hline estado 2 vs estado 5 (repetición 1) & 51 & 29,98 & 18,73 & Significativa \\
\hline estado 3 vs estado 4 (repetición 1) & 51 & 0,853 & 18,73 & NS \\
\hline estado 3 vs estado 5 (repetición 1) & 51 & 36,03 & 18,73 & Significativa \\
\hline estado 4 vs estado 5 (repetición 1) & 51 & 36,89 & 18,73 & Significativa \\
\hline estado 1 vs estado 2 (repetición 2) & 51 & 1,206 & 18,73 & NS \\
\hline estado 1 vs estado 3 (repetición 2) & 51 & 45,07 & 18,73 & Significativa \\
\hline estado 1 vs estado 4 (repetición 2) & 51 & 97,81 & 18,73 & Significativa \\
\hline estado 1 vs estado 5 (repetición 2) & 51 & 11,01 & 18,73 & NS \\
\hline estado 2 vs estado 3(repetición 2) & 51 & 43,86 & 18,73 & Significativa \\
\hline estado 2 vs estado 4 (repetición 2) & 51 & 96,61 & 18,73 & Significativa \\
\hline estado 2 vs estado 5 (repetición 2) & 51 & 9,804 & 18,73 & NS \\
\hline estado 3 vs estado 4 (repetición 2) & 51 & 52,75 & 18,73 & Significativa \\
\hline estado 3 vs estado 5 (repetición 2) & 51 & 34,06 & 18,73 & Significativa \\
\hline estado 4 vs estado 5 (repetición 2) & 51 & 86,81 & 18,73 & Significativa \\
\hline
\end{tabular}


No se observó un comportamiento agresivo por parte de las hormigas. La respuesta de alarma puede variar de una especie a otra, en algunas sirve para bajar el umbral de ataque, en otras ayuda a orientar al individuo al lugar donde se origina el peligro y en las especies más dóciles provoca un comportamiento de escape (Hanson \& Gauld, 2006). La defensa de las plantas asociadas a las hormigas no siempre sucede, algunas hormigas nectívoras pueden alimentarse tanto del néctar de la planta como de sustancias dulces producidas por insectos. (Rico \& Morais, 2006). No se considera de beneficio para Heliconia rostrata la presencia de Linepithema dispertitum ya que durante la investigación se observó en asociación con Pseudococcidae, según Ramos \& Serna (2004) algunos integrantes de Pseudococcidae se consideran una plaga de importancia para la agricultura.

La actividad de la hormiga en las inflorescencias varió durante el día en ambas repeticiones, la actividad más baja se observó durante la mañana, mientras que en la noche la actividad fue mayor; la actividad de las hormigas depende principalmente de la permanencia del recurso y de su preferencia en su medio y los ámbitos óptimos de diferencias ambientales. Estudios realizados en Camponotus indican una mayor actividad durante las horas de la noche, esto coincide con la producción máxima de néctar del cactus con el que se asocia (Novoa, Redolfi \& Ceroni, 2005). En laboratorio se ha observado a Crematogaster scutellaris con actividad baja durante la mañana, para luego aumentar hasta las 16:00h y descender posteriormente, mientras que en Tapinoma tigerrima con actividad mayor en las primeras horas de la mañana y decreciendo durante el día (Redolfi et al., 2003).

En cuanto a las preferencias de Linepithema dispertitum hacia el estado de inflorescencia, con los promedios más bajos de hormigas en las inflorescencias con menor cantidad de alimento disponible y con brácteas marchitas. Se sabe que las hormigas pueden cambiar su patrón de colecta de néctar, reaccionan sensiblemente a las diferencias en el néctar, son capaces de seleccionar y preferir soluciones azucaradas que contengan una compleja mezcla de aminoácidos, puede discriminar entre baja y alta calidad (Rico \& Moráis, 2006). Los promedios más altos de hormigas estuvieron en las inflorescencias con mayor número de brácteas y sin brácteas marchitas, lo que sugiere una mayor disponibilidad de alimento y un néctar más atractivo para la hormiga. Cambios en las plantas pueden inducir a que esta produzca néctar en mayor cantidad y de mejor calidad, lo que incrementa la atracción de la planta hacia las hormigas, lo que a su vez provoca un aumento del patrullaje en la fuente de alimento, no obstante las hormigas también pueden elegir consumir un alimento del que hay poco, no necesariamente coinciden con los más abundantes en la dieta (Farji-Brener et al., 1992).

\section{AGRADECIMIENTOS}

A mi familia por todo su apoyo. A Ana Teresa Garita Salas por toda su colaboración y apoyo durante la realización de esta investigación.

\section{REFERENCIAS}

Alatorre-Bracamontes, C., Vázquez-Bolaños, M. (2010). Lista comentada de las hormigas (Hymenoptera: Formicidae) del norte de México. Dugesiana. 17(1), 9-36.

Farji-Brener, A. G., Folgarait, P. \& Protomastro, J. (1992). Asociación entre el arbusto Capparis retusa (Capparidaceae) y las hormigas Camponotus blandus y Acromyrmex striatus (Hymenoptera: Formicidae). Revista de Biología Tropical. 40(3), 341-344.

Jerez, E. (2007). El cultivo de las Heliconias. Cultivos tropicales 28(1), 29-35.

Guitián, P., Navarro, L. \& Guitián, J. (1995). Efecto de la extracción en la producción de néctar en flores de Melittis melissophyllum (Labiatae). Anales de Jardín Botánico de Madrid. 52(2), 163-169.

Hanson, P.E., Gauld, I.D. (Eds.). (2006). Hymenoptera de la Región Neotropical. Memoirs of the American Entomological Institute 77.

Novoa, S., Redolfi, I. \& Ceroni, A. (2005). Patrones de actividad diario de la hormiga Camponotus sp. en los botones florales del cactus Neoraimondia arequipensis subsp. roseiflora (Werdermann \& Backeberg) Ostolaza. Ecología Aplicada. $4(1,2), 77-81$.

Ramos, A.P., Serna, F.C. (2004). Coccoidea de Colombia, con énfasis en las cochinillas harinosas (Hemiptera: Pseudococcidae). Revista Facultad Nacional de Agronomía Medellin. 57 (2), 2383-2412.

Ramírez, M., Chacon, P., Armbrecht, I. \& Calle, Z. (2001). Contribución al conocimiento de las interacciones entre plantas, hormigas y homópteros en bosques secos de Colombia. Caldasia. 23(2), 523-536.

Redolfi, I., Tinaut, A., Pascual, F. \& M.Campos. (2003). Patrón de actividad de Tapinoma nigerrima (Nylander) y Crematogaster scutellaris (Oliver) (Hymenoptera, Formicidae) en el cultivo de olivo y en el laboratorio. Zoología Baetica.14, 37-55.

Rico, V.G., Morais, H.C. (2006). Efecto de una fuente de alimentación experimental sobre una asociación Hormiga-Hemíptero. Acta Zoológica Mexicana. 22(3), 23-28.

Wild, A.L. (2007). Taxonomic Revision of the Ant Genus Linepithema (Hymenoptera: Formicidae). University of California Press: Berkeley, CA, EE.UU. 126, 1-162. 
